\title{
Five-Fold Helically Corrugated Waveguide for High-Power W-Band Gyro-Devices and Pulse Compression
}

\author{
Craig R. Donaldson, Liang Zhang, Peter Hiscock, Michael Harris, Matthew J. Beardsley, Peter G. Huggard, \\ Colin G. Whyte, Adrian W. Cross, and Wenlong He
}

\begin{abstract}
The design, simulation, manufacture and measurement of a W-band five-fold ( $5 \mathrm{~F}$ ) helically corrugated waveguide (HCW) is reported. The $5 \mathrm{~F} \mathrm{HCW}$ is based on the coupling of the traveling $\mathrm{TE}_{31}$ and near cut-off $\mathrm{TE}_{22}$ modes to create an operating eigenwave. The fabricated test structure has circular waveguide ports and features elliptical polariser sections and broadband $T E_{11}$ to $T E_{31}$ mode converters on either side of the $5 \mathrm{~F} \mathrm{HCW}$. The optimised mode converter design, based on a four-fold (4F) HCW, has a predicted power conversion efficiency greater than $90 \%$ from 89 to $102.5 \mathrm{GHz}$, and $96 \%$ peak efficiency at $94 \mathrm{GHz}$. The optimization of the $5 \mathrm{~F} \mathrm{HCW}$ geometry produced an eigenwave suitable for gyro-devices, but the optimization could equally well have been directed to applications such as pulse compression and microwave undulators. Analysis of simulated electric field profiles showed that the propagating power in the $5 \mathrm{~F} \mathrm{HCW}$ was increased by a factor of 6 over that in the 3F HCW at equivalent peak electric field strength. This is due to the larger diameter of the waveguide. Test structures were manufactured through a combination of precision machining of a sacrificial mandrel, copper growth by electroforming followed by removal of the aluminium mandrel by chemically etching. Measurements of the $5 \mathrm{~F}$ HCW structure's dispersion showed excellent agreement with the prediction over the design range of 90 to $98 \mathrm{GHz}$.
\end{abstract}

Index Terms-W-band; mode converter; helically corrugated waveguide; precision manufacture

\section{INTRODUCTION}

$\mathbf{H}$ ELICALLY corrugated waveguides (HCW) have unique dispersive properties that make them attractive for a number of millimetre wave applications. In 1998 it was proposed [1] that a waveguide with a helical corrugation on its inner surface could resonantly couple two waves, one close to cut-off and one far from cut-off, to create an operating

This work was supported by the Engineering and Physical Sciences Research Council (EPSRC) U.K. under Research Grant EP/K029746/1, EP/M008622/1 and EP/S00968X/1 and Science and Technology Facilities Council (STFC) U.K. under Research Grant ST/T003227/1

C. R. Donaldson, L. Zhang, C. G. Whyte, and A. W. Cross are with the Scottish Universities Physics Alliance, Department of Physics, University of Strathclyde, Glasgow G4 ONG, U.K, and are also members of the Cockcroft Institute, Warrington Bank Quay WA4 4AD, UK.

P. Hiscock, M. Harris, M. J. Beardsley, and P. G. Huggard are with RAL Space, STFC Rutherford Appleton Laboratory, Didcot, OX11 0QX, U.K.

W. He is with College of Electronics and Information Engineering, Shenzhen University, Shenzhen 518060, China. eigenwave. Through optimization of the corrugation geometry the eigenwave can be tailored to provide properties well suited to the coupling of electron beams and electromagnetic waves in gyro-devices [2]-[6]. A dispersion profile with a constant group velocity in the region of small wave numbers can be synthesised which allows effective coupling to an electron beam. With a suitably high group velocity, either broadband amplification or wide band oscillation can be achieved, operation in the region of low wave numbers reduces sensitivity to velocity spread in the electron beam and provides high efficiency. In the field of pulse compression a dispersion curve with a monotonic group velocity and low losses far from cutoff is desired. This can be provided by an optimised HCW [7]-[10]. The HCW can be further utilized in microwave undulators [11], [12] where a backward wave can be excited by a traveling wave to interact with the electron bunch and significantly enhance the effective interaction length.

The general HCW has both azimuthal and axial corrugations. Its surface is described through the following relation, in polar cylindrical coordinates, $r(\theta, z)=r_{0}+r_{1} \cos \left(m_{B} \theta+\right.$ $2 \pi z / d)$, where $r_{0}=$ average radius, $r_{1}=$ corrugation amplitude, $d=$ corrugation period and $m_{B}$ is the fold number. The corrugation twist direction is also denoted as ether lefthand $(\mathrm{LH})$ or right-hand $(\mathrm{RH})$ with respect to the direction of propagation.

Coupling between modes takes place when the synchronism conditions $k_{1} \pm k_{2}=2 \pi / d$ and $m_{1} \pm m_{2}=m_{B}$, where $k$ is the axial wave number, $m_{x}$ are the azimuthal index and subscripts 1 and 2 denote the modes, are met. In a three-fold (3F) HCW [4], [13] the coupled modes are usually the travelling $\mathrm{TE}_{11}$ and $\mathrm{TE}_{21}$ waves. In a five-fold $(5 \mathrm{~F}) \mathrm{HCW}$ many options exist. We report a design coupling the travelling $\mathrm{TE}_{31}$ and $\mathrm{TE}_{22}$ waves. The choice of coupled modes allowed this $5 \mathrm{~F} \mathrm{HCW}$ to demonstrate that the key straight dispersion line characteristics useful for gyro-devices can be designed and measured. The mode converter used to generate the $\mathrm{TE}_{31}$ mode has broader applicability, for instance to pulse compression.

The use of higher order modes in the $5 \mathrm{~F} \mathrm{HCW}$ results in a waveguide with a larger diameter than a comparable $3 \mathrm{~F}$ $\mathrm{HCW}$ for a given frequency of operation, enabling a more powerful electron beam to be propagated through the structure as well as a higher maximum wave power before the onset of breakdown. Although its potential applications are wider, 
the $5 \mathrm{~F} \mathrm{HCW}$ reported in literature to date has primarily been applied to pulse compression at X-band [8], [9] and Ka-band [10] frequencies. In these cases the unique group velocity profile of the $\mathrm{HCW}$ eigenwave has been engineered to compress an $\mathrm{X}$-band pulse to a very high peak power of $3 \mathrm{GW}[9]$.

A $3 \mathrm{~F} \mathrm{HCW}$ has been fabricated and measured at frequencies up to $372 \mathrm{GHz}$ [13]. An eight-fold (8F) $\mathrm{HCW}$ in the same $372 \mathrm{GHz}$ frequency range [14] increased the possible power handling while reducing the sensitivity to electron beam optics but has a higher level of mode competition and narrower band components. The $8 \mathrm{~F} \mathrm{HCW}$ allows the opportunity to explore the operation at a higher order mode. However, previous experience on the manufacture of an $8 \mathrm{~F} \mathrm{HCW}$ operating at $372 \mathrm{GHz}$ indicates a challenge to achieve good surface finish due to the high periodic number in the azimuthal coordinate, which leads to a relatively high transmission loss although the dispersion curve is close to the theoretical prediction. A lower periodic number, such as the $5 \mathrm{~F}$, will be a good balance between the manufacturing capability and the operating mode which are more realistic to meet the practical applications that require high power, broadband operation: it was previously proposed for W-band gyro-devices [15].

This paper presents the design, precision manufacturing approach and measurement of a W-band 5F HCW designed for use at $94 \mathrm{GHz}$ with around $10 \%$ bandwidth, $94 \mathrm{GHz}$ is a key, atmospheric window, frequency for radars and communications applications. In order to characterise the $5 \mathrm{~F} \mathrm{HCW}$, linear to circular polarisers and mode converters are required to convert the fundamental $\mathrm{TE}_{11}$ modes of the input and output cylindrical waveguides to the required $\mathrm{TE}_{31}$ mode. The conversion of the $\mathrm{TE}_{11}$ mode to higher order modes has been extensively studied, including conversion to the $\mathrm{TE}_{01}$ [16], [17], $\mathrm{TM}_{01}$ [18], [19], $\mathrm{HE}_{11}$ [20], [21] modes and many others. A four-fold (4F) $\mathrm{HCW}$, coupling $\mathrm{TE}_{11}$ to $\mathrm{TE}_{31}$ modes, can achieve the efficiency required over a sufficiently broadband frequency range. Such couplers have been experimentally verified at $\mathrm{X}$-band [8]. The design of a compact, i.e. short, $4 \mathrm{~F}$ $\mathrm{HCW}$ has additional complexity through the increase of the diameter needed to transition from regular circular waveguide to the larger $5 \mathrm{~F} \mathrm{HCW}$ waveguide.

\section{DESIGN}

Figure 1 presents the polarisation and mode conversion elements required for characterisation of the W-band 5F HCW. Starting with a linear polarisation propagating in circular, fundamental mode waveguide, the propagating mode $\mathrm{TE}_{11}$ mode is first converted to circular polarisation and then to $\mathrm{TE}_{31}$ in a $4 \mathrm{~F} \mathrm{HCW}$ mode converter. The wave then passes through the $5 \mathrm{~F} \mathrm{HCW}$, enters a second, identical $4 \mathrm{~F} \mathrm{HCW}$ and then is converted back to linear polarisation. Designing the structure with linearly polarised inputs and outputs facilitates measurement using a vector network analyser (VNA).

An elliptically shaped polariser type was chosen as it could be manufactured at the same time using the same method as the $\mathrm{HCW}$ sections. The polariser configuration is simple with the circular waveguide linearly converting to an ellipse.

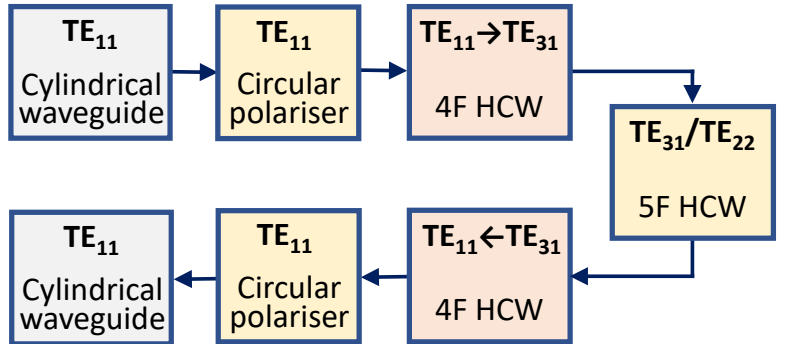

Fig. 1: Schematic diagram showing the required components to measure a $5 \mathrm{~F} \mathrm{HCW}$.

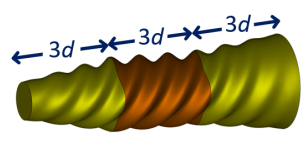

(a) $4 \mathrm{~F} \mathrm{HCW}$

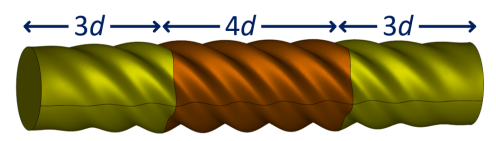

(b) $5 \mathrm{~F} \mathrm{HCW}$
Fig. 2: Illustrations of the $4 \mathrm{~F}$ and $5 \mathrm{~F} \mathrm{HCW}$, as displayed in CST-MS. The up- and down-tapers are coloured in olive green and the uniform corrugation section in brown.

The ellipse major axis angle is set at $\pm 45^{\circ}$ to the polarisation of the circular waveguide, depending on the desired rotation direction of the circularly polarised waves. The optimised elliptical geometry had a major axis of $3.00 \mathrm{~mm}$, a minor axis of $2.60 \mathrm{~mm}$ and total length of $18.76 \mathrm{~mm}$.

The design of the 4F HCW corrugation geometry considers the dispersion of the two coupled modes $\left(\mathrm{TE}_{11}\right.$ and $\left.\mathrm{TE}_{31}\right)$ which is calculated through coupled-mode theory [22]. The intersection point of the modes, theoretically, has complete conversion from one to the other. The overall length of guide is a tradeoff between required bandwidth and maximum conversion efficiency. A short $\mathrm{HCW}$ will have a wide bandwidth but lower efficiency. The $4 \mathrm{~F} \mathrm{HCW}$ was optimized for a central frequency of $94 \mathrm{GHz}$ with corrugation parameters of $r_{0}=2.26 \mathrm{~mm}, r_{1}=0.28 \mathrm{~mm}, d=2.12 \mathrm{~mm}$, length $3 d$ with LH twist. Corrugation tapers of length $3 d$ lead in and out of the central region.

The left hand $4 \mathrm{~F} \mathrm{HCW}$ is illustrated in Fig. 2a. It is comprised of the uniform HCW section with a linear taper, corrugated to circular cross-section, before (pre-taper) and after (post-taper). Each taper includes a linear tapering of the average radius to match the waveguides on either side of the assembly. The pre-taper initial radius was $1.3 \mathrm{~mm}$ while the post-taper radius increased to a final value of $3.24 \mathrm{~mm}$ : both tapers have a length of $3 d$.

The simulation of the $4 \mathrm{~F} \mathrm{HCW}$ mode converter, shown in Fig. 3, was performed using CST Microwave Studio (CSTMS). A circularly polarised $\mathrm{RH} \mathrm{TE}_{11}$ mode was the input source and a maximum conversion efficiency of $96 \%$ at $94 \mathrm{GHz}$ to the $\mathrm{LH} \mathrm{TE}_{31}$ mode was predicted. An achieved bandwidth of $89-102.5 \mathrm{GHz}$ with higher than $90 \%$ transmission $\left(\mathrm{S}_{21}>-0.4 \mathrm{~dB}\right)$ and reflection, $\mathrm{S}_{11}$, below $-29 \mathrm{~dB}$, met design targets.

The RH 5F HCW is illustrated in Fig. 2b. The LH TE 31 and $\mathrm{RH} \mathrm{TE}_{22}$ modes are coupled and the calculated dispersion is 


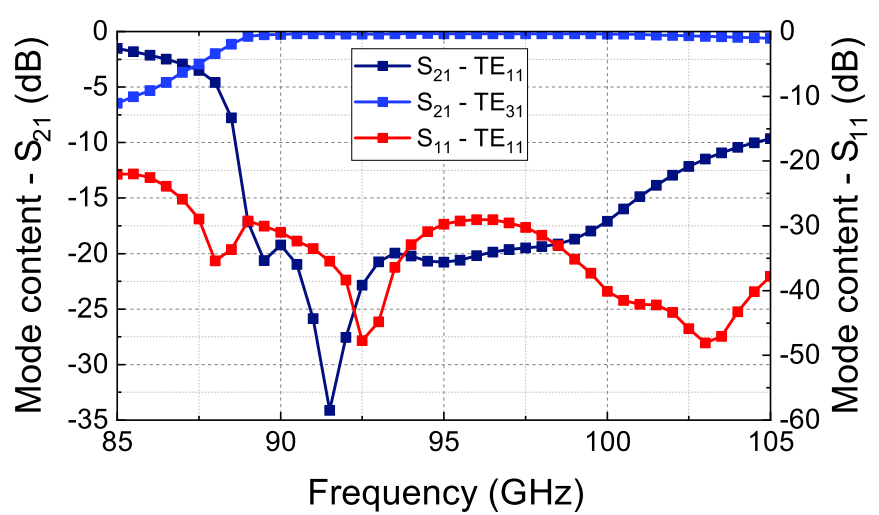

Fig. 3: Simulation of the LH 4F HCW mode converter with an input RH TE 11 mode.

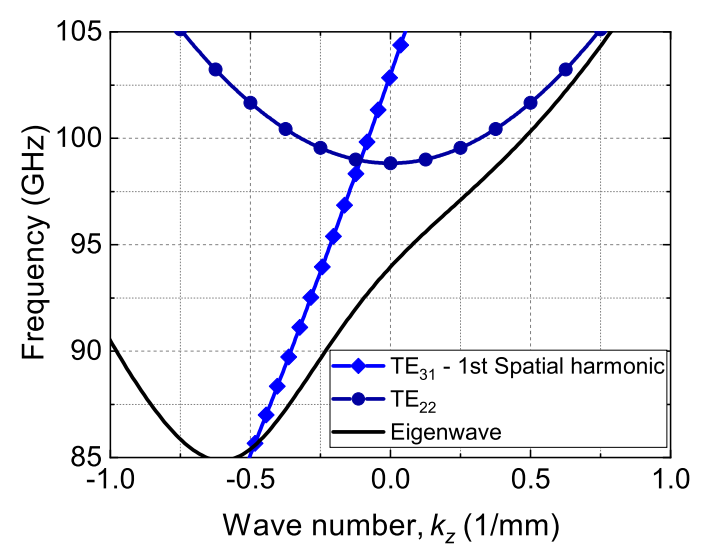

Fig. 4: Dispersion diagram for the RH 5F HCW.

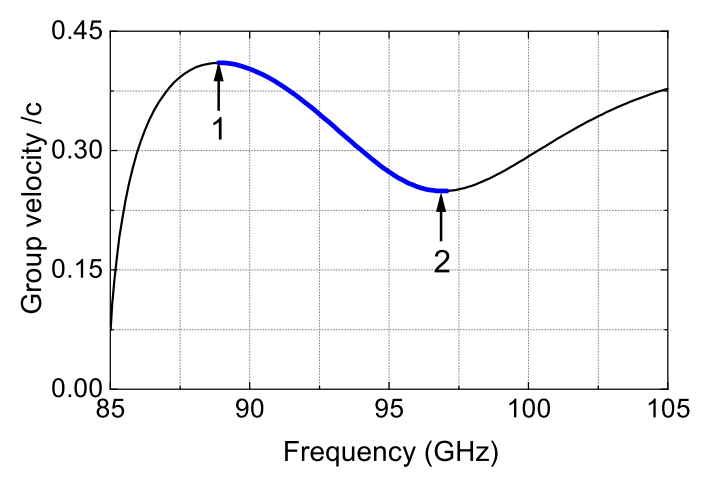

Fig. 5: The group velocity of the calculated eigenwave in the $\mathrm{RH} 5 \mathrm{~F} \mathrm{HCW}$ with frequency range suitable for pulse compression highlighted in blue.

given in Fig. 4. The corrugation geometry is $r_{0}=3.24 \mathrm{~mm}$, $r_{1}=0.37 \mathrm{~mm}, d=3.65 \mathrm{~mm}$, length $4 d$ and RH twist. The choice of length allowed measurement of the dispersion properties and transmission/reflection while keeping the overall length of the part within the constraints of the machining process. On each side of the uniform HCW section are preand post-tapers, both $3 d$ long, with a uniform tapering of their corrugation amplitude.

Pulse compression can be achieved when a microwave pulse with a suitable frequency chirp propagates through a $5 \mathrm{~F} \mathrm{HCW}$.

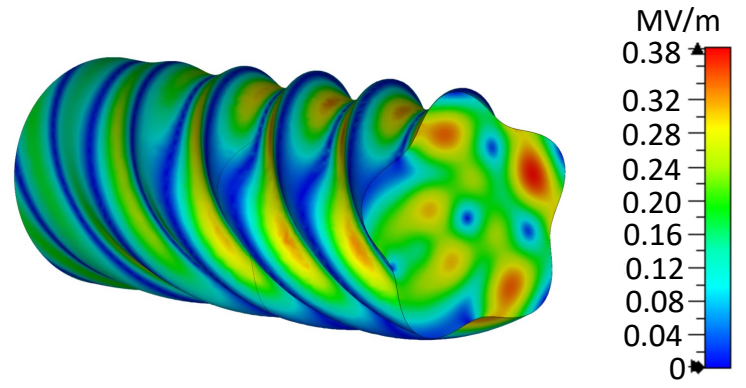

Fig. 6: Colour coded electric field pattern at a cross-section of the RH $5 \mathrm{~F} \mathrm{HCW}$ with an input $1 \mathrm{~kW} \mathrm{TE}_{31}$ mode at $94 \mathrm{GHz}$.

If the input pulse has frequency content that is tailored such that the start of the pulse contains frequencies which have low group velocities while frequencies experiencing higher group velocities occur later in the pulse, it can be arranged that all frequencies arrive at the waveguide exit at close to the same time. This results in pulse length compression with a corresponding increase in pulse peak amplitude. Long lengths of waveguide may be required to achieve significant compression ratios.

Fig. 5, shows that, in the $5 \mathrm{~F} \mathrm{HCW}$ eigenwave, a viable frequency range exists from 87 to $97 \mathrm{GHz}$ where the group velocity drips monotonically from $0.41 \mathrm{c}$ to $0.25 \mathrm{c}$.

The ratio of the output to the input magnitude, the compression ratio $(K)$ can be calculated [8] using $K=\Delta f L\left(1 / v_{g 1}-\right.$ $\left.1 / v_{g 2}\right) e^{-\xi L}$ where $\Delta f$ is the frequency range between points 1 and $2, v_{g 1}$ and $v_{g 2}$ are the corresponding group velocities, $L$ is the length of the HCW section and $\xi$ is a loss factor. Not considering losses, a compression ratio of $\sim 16.4$ is predicted for a uniform HCW section length of $86 d$.

The electric field mode pattern of the eigenwave within a cross-section of the 5F HCW is shown in Fig. 6. To prevent breakdown, the maximum electric field at the waveguide walls cannot exceed $\mathrm{E}=3 \mathrm{MV} / \mathrm{m}$ for a continuous wave $(\mathrm{CW})$ in air. The calculated power handling of the $5 \mathrm{~F} \mathrm{HCW}$ operating at W-band in this case is $62 \mathrm{~kW}, 6$ times higher than the comparable $3 \mathrm{~F} \mathrm{HCW} \mathrm{[3].} \mathrm{At} \mathrm{high} \mathrm{vacuum} \mathrm{the} \mathrm{power} \mathrm{handling}$ would be larger.

\section{MANufacture}

The manufacture of the hollow copper HCW's is challenging. Their small aperture and feature size, with tight geometric tolerance and high surface quality requirements, precludes direct machining, therefore electroforming onto a sacrificial structure is a natural choice. A negative of the waveguide, the mandrel, is machined into an aluminium alloy, grade 6082, rod. A high speed precision computer numerical control (CNC) milling machine, the KERN Micro mill, is used to produce the shape. During the machining process, the aluminium rod is first turned on a lathe to the required cylindrical profile. It is then transferred to the milling machine and physically supported along its length as a ball nosed cutter removes the unwanted material via a repeat series of small axial cuts [13], [14]. This delivers the required profile to an accuracy of \pm a few $\mu \mathrm{m}$ with a very high quality surface finish: photographs 


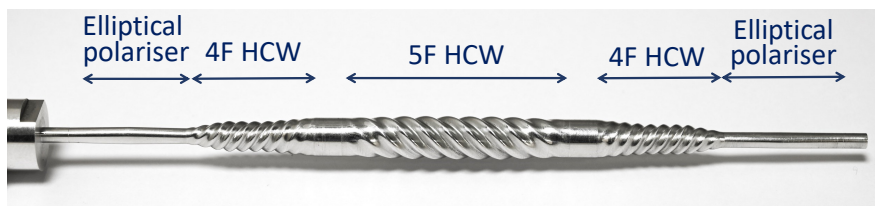

Fig. 7: Photograph of the aluminium former. The polarisation reference flat can be seen at the left hand edge of the image.

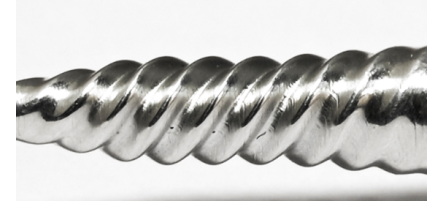

(a) $4 \mathrm{~F} \mathrm{HCW}$

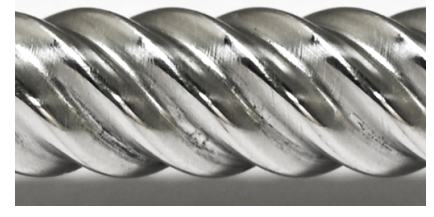

(b) $5 \mathrm{~F} \mathrm{HCW}$
Fig. 8: Closer pictures of the two helical waveguide surfaces taken during inspection of their surface finish. The external diameters are $5.08 \mathrm{~mm}$ (a) and $7.22 \mathrm{~mm}$ (b)

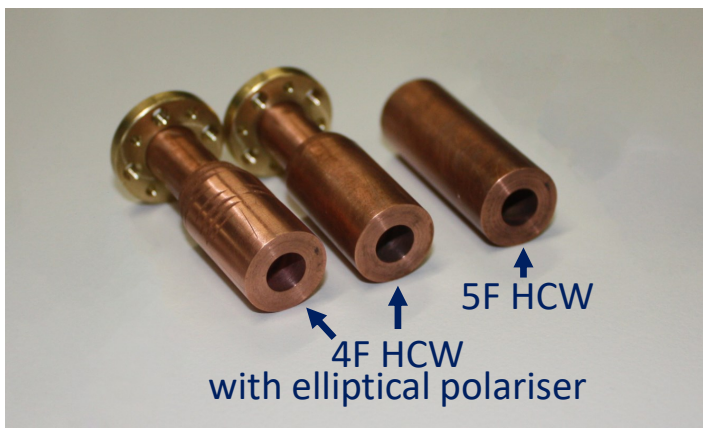

Fig. 9: Photograph of the 4F and 5F HCWs.

of the completed mandrel are shown in Fig. 7 and 8 . No post machining polishing is required.

Copper is then electrodeposited onto the mandrel until the desired wall thickness of $4 \mathrm{~mm}$ was reached. The aluminium is subsequently removed in a $\mathrm{NaOH}$ solution to leave the copper positive waveguide. Conventional machining is used to shape the exterior of the waveguide device. The orientation of the elliptical polarisers are known due to the provision of a flat surface on the mandrel, as shown on one side of Fig. 7. This datum area was not coated in copper, and its inclusion allows accurate alignment of standard UG387/m waveguide flanges: Fig. 9.

\section{MeAsurement}

An Anrisu MS4647A VNA with 75-110 GHz frequency extension heads was used to measure the microwave properties of the $4 \mathrm{~F}$ and $5 \mathrm{~F} \mathrm{HCW}$ 's. The measurement setup is shown in Fig. 10. The VNA ports are rectangular waveguides with linear polarisation so standard rectangular to circular waveguide adapters were required. An aluminium sleeve was used to align the end faces of the two $4 \mathrm{~F} \mathrm{HCWs}$ in the first measurement and then the $5 \mathrm{~F} \mathrm{HCW}$ to each $4 \mathrm{~F} \mathrm{HCW}$ for characterisation of the latter.

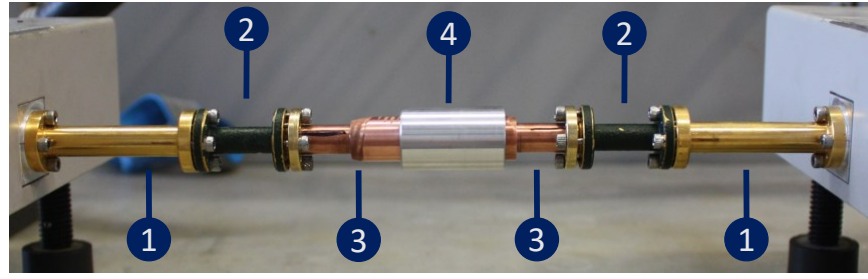

(a) Set-up for measurement of S-parameters without the 5F HCW

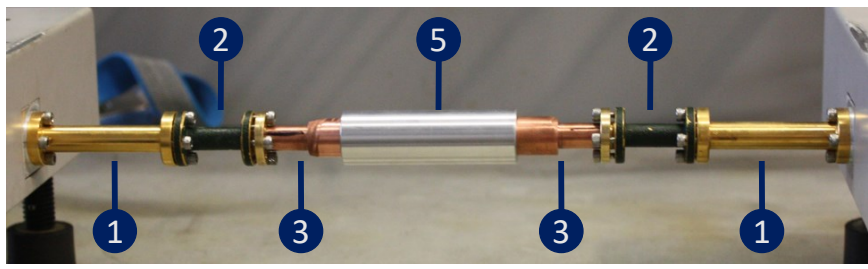

(b) Set-up for measurement of S-parameters including the 5F HCW

Fig. 10: Photo of the measurement of the 5F HCIR on a vector network analyser showing: 1. VNA port extenders, 2. rectangular to circular waveguide adapters, 3. a $4 \mathrm{~F} \mathrm{HCW}$ containing elliptical polariser, 4 . aluminium sleeve to align the contacting faces of the $4 \mathrm{~F} \mathrm{HCW}$ and 5 . an aluminium sleeve covering the $5 \mathrm{~F} \mathrm{HCW}$.

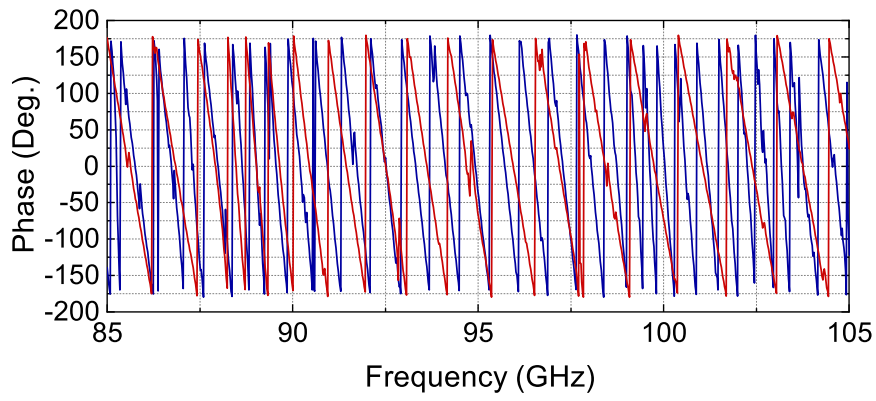

Fig. 11: The measurement data of the phase evolution through the waveguide components shown in Fig. 10a (Red line) and Fig. 10b (blue line).

The dispersion of the $5 \mathrm{~F} \mathrm{HCW}$ was calculated through two measurements. In the first, Fig. 10a, the phase evolution through the waveguides was measured. Then in the second, Fig. 10b, the 5F HCW was included and the phase was remeasured. The subtraction of the two phase curves, which are shown in Fig. 11, and dividing by the length of the 5F HCW allowed calculation of $k_{z}$. The measurement was repeated for both alignments of the elliptical polarisers $\left( \pm 45^{\circ}\right)$ so that the circularly polarised waves entering the $4 \mathrm{~F} \mathrm{HCW}$ were in either left- or right-hand rotation. Simulated dispersion, from CST-MS, used the same method of phase calculation and the same components so that effects from non-ideal mode conversion are included. The measurement is displayed in Fig. 12. There is good agreement between the measured and calculated eigenwave dispersion between $\sim 89$ and 98 GHz. Above this range coupling to higher order modes, not considered in the coupled mode theory calculations, becomes possible. Above $98 \mathrm{GHz}$ the eigenwave line calculated by coupled mode theory can no longer be considered accurate and 


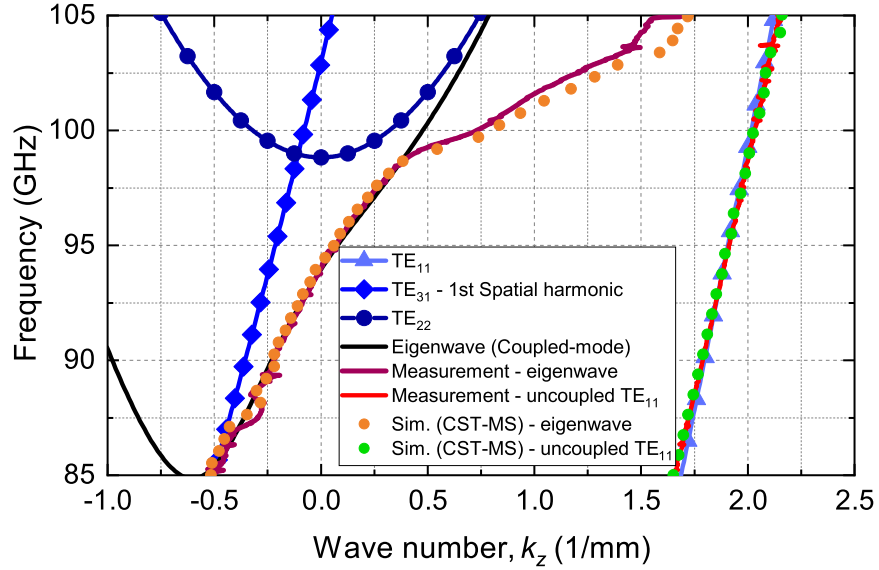

Fig. 12: Dispersion diagram for the RH $5 \mathrm{~F}$ HCW including the calculated waveguide modes, eigenwave and the measured data.

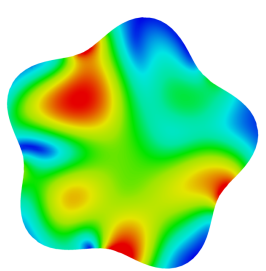

(a) $85 \mathrm{GHz}$

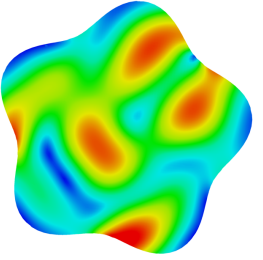

(b) $95 \mathrm{GHz}$

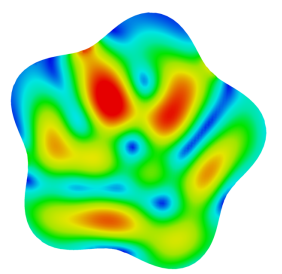

(c) $105 \mathrm{GHz}$
Fig. 13: Simulated electric field at a cross-section of the $\mathrm{RH}$ $5 \mathrm{~F} \mathrm{HCW}$ replicating the components used in the measurement.

it rapidly diverges from both the measured and CST simulated dispersions. Below $89 \mathrm{GHz}$ the distortion of the phase seen is most likely caused by residual lower order mode content due to the reduction in the mode converter efficiency and is to be expected from the results shown in Fig. 3. The simulated electric field patterns in the $5 \mathrm{~F} \mathrm{HCW}$ at the centre frequency and both extremes is shown in Fig. 13. When the polarisers were in the opposite rotation, there was no mode conversion in the $4 \mathrm{~F} \mathrm{HCW}$ and so the measured dispersion aligned with the $\mathrm{TE}_{11}$ waveguide mode as expected.

The S-parameters of the components pictured in Fig. 10b were measured and are presented in Fig. 14. The transmission dips and peaks in the reflection are attributable to the back-to-back measurement, which causes a small degree of energy trapping as discussed in [23], [24] and also evidenced in lower frequency $5 \mathrm{~F} \mathrm{HCW}$ measurements [8]. The phase measurements, shown in Fig. 11, have local distortions that are attributable to the same trapping. When the phase data is converted to dispersion these small features are obscured resulting in a smooth profile. Over the $90-98 \mathrm{GHz}$ frequency range the average transmission loss was $1.05 \mathrm{~dB}$ with an average reflection of $-25 \mathrm{~dB}$, when the sharp features are reasonably accounted for. The loss value includes a contribution from the well characterised rectangular to circular adapters which had a combined average loss of $0.3 \mathrm{~dB}$.

In this frequency range surface roughness in combination with the purity of the electroformed copper make a signif-

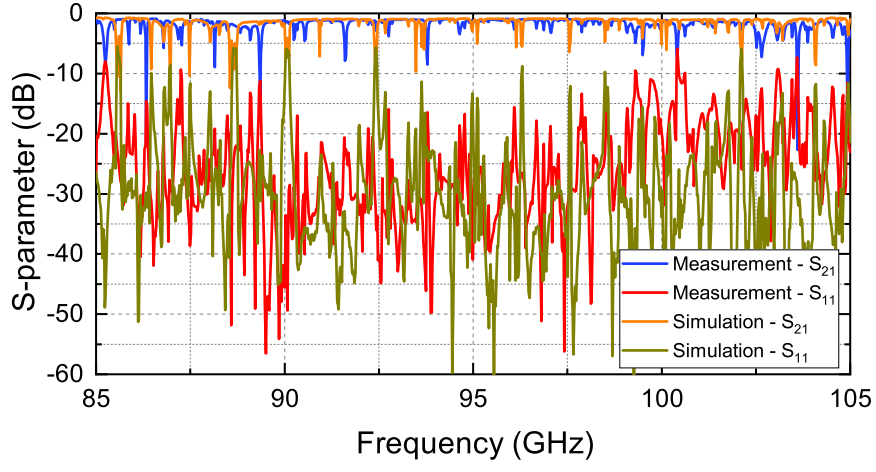

Fig. 14: Measurement and simulation of the transmission and reflection through the $\mathrm{LH} 4 \mathrm{~F}$ and $\mathrm{RH} 5 \mathrm{~F}$ HCWs when the circular polarisers output the $\mathrm{RH} \mathrm{TE}_{11}$ mode.

icant contribution to material conductivity. The component circuit was simulated over a range of conductivities to find the best agreement between measurement and prediction. A conductivity of $3 \times 10^{7} \mathrm{~S} / \mathrm{m}$, about half the value of smooth oxygen free high conductivity (OFHC) copper, had a good match to experimental measurements, the measured values are consistent with a very high quality machined surface.

\section{CONCLUSION}

This paper presents the design and measurement of a Wband $\mathrm{RH} 5 \mathrm{~F} \mathrm{HCW}$. The measurement results show excellent agreement with the analytically calculated eigenwave over the frequency range $90-98 \mathrm{GHz}$. The $\mathrm{TE}_{11}$ to $\mathrm{TE}_{31}$ mode converter, which was a LH $4 \mathrm{~F} \mathrm{HCW}$, was optimized through simulation to have a bandwidth of $89-102 \mathrm{GHz}$ with greater than $90 \%$ conversion efficiency ( $96 \%$ max). The transmission measurement of the components showed that the conductivity of the parts was about half of that for OFHC copper and the close matching of the measured wave dispersion showed that the geometry was precisely made. This work facilitates future study for the application of the $5 \mathrm{~F} \mathrm{HCW}$ in millimetre-wave gyro-devices and pulse compressors.

\section{REFERENCES}

[1] G. G. Denisov, V. L. Bratman, A. D. R. Phelps, and S. V. Samsonov, "Gyro-TWT with a helical operating waveguide: new possibilities to enhance efficiency and frequency bandwidth," IEEE Trans. Plasma Sci., vol. 26 , no. 3 , pp. 508-518, 1998, doi: 10.1109/27.700785.

[2] V. L. Bratman, A. W. Cross, G. G. Denisov, W. He, A. D. R. Phelps, K. Ronald, S. V. Samsonov, and A. R. Young, "High-gain wideband gyrotron traveling wave amplifier with a helically corrugated waveguide," Phys. Rev. Lett., vol. 84, no. 12, pp. 2746-2749, 2000 , doi: 10.1103/PhysRevLett.84.2746.

[3] W. He, C. R. Donaldson, L. Zhang, K. Ronald, A. D. R. Phelps, and A. W. Cross, "Broadband amplification of low-terahertz signals using axis-encircling electrons in a helically corrugated interaction region," Phys. Rev. Lett., vol. 119, 184801, 2017, doi: 10.1103/PhysRevLett.119.184801.

[4] W. He, C. R. Donaldson, L. Zhang, P. McElhinney, K. Ronald, and A. W. Cross, "High power wideband gyrotron backward wave oscillator operating towards the terahertz region," Phys. Rev. Lett., vol. 110, no. 16, 165101, 2013, doi: 10.1103/PhysRevLett.110.165101.

[5] S. V. Samsonov, G. G. Denisov, I. G. Gachev, and A. A. Bogdashov, "CW operation of a W-band high-gain helical-waveguide gyrotron traveling-wave tube," IEEE Electron Device Lett., vol. 41, no. 5, pp. 773-776, 2020, doi: 10.1109/LED.2020.2980572. 
[6] S. V. Samsonov, I. G. Gachev, G. G. Denisov, A. A. Bogdashov, S. V. Mishakin, A. S. Fiks, E. A. Soluyanova, E. M. Tai, Y. V. Dominyuk, B. A. Levitan, and V. N. Murzin, "Ka-band gyrotron traveling-wave tubes with the highest continuous-wave and average power," IEEE Trans. Electron Devices, vol. 61, no. 12, pp. 4264-4267, 2014, doi: 10.1109/TED.2014.2364623.

[7] S. V. Samsonov, A. D. R. Phelps, V. L. Bratman, G. Burt, G. G. Denisov, A. W. Cross, K. Ronald, W. He, and H. Yin, "Compression of frequencymodulated pulses using helically corrugated waveguides and its potential for generating multigigawatt rf radiation," Phys. Rev. Lett., vol. 92, 118301, 2004, doi: 10.1103/PhysRevLett.92.118301.

[8] L. Zhang, S. V. Mishakin, W. He, S. V. Samsonov, M. McStravick, G. D. Denisov, A. W. Cross, V. L. Bratman, C. G. Whyte, C. W. Robertson, A. R. Young, K. Ronald, and A. D. R. Phelps, "Experimental study of microwave pulse compression using a five-fold helically corrugated waveguide," IEEE Trans. Microw. Theory Techn., vol. 63, no. 3, pp. 1090-1096, 2015, doi: 10.1109/TMTT.2015.2393882.

[9] V. L. Bratman, G. G. Denisov, N. G. Kolganov, S. V. Mishakin, S. V. Samsonov, A. W. Cross, W. He, L. Zhang, M. McStravick, C. G. Whyte, A. R. Young, K. Ronald, C. W. Robertson, and A. D. R. Phelps, "Generation of $3 \mathrm{GW}$ microwave pulses in X-band from a combination of a relativistic backward-wave oscillator and a helicalwaveguide compressor," Phys. Plasmas, vol. 17, 110703, 2010, doi: $10.1063 / 1.3505825$.

[10] L. A. Yurovskiy, I. V. Zotova, N. S. Ginzburg, M. N. Vilkov, R. M. Rozental, S. V. Samsonov, and E. B. Abudakirov, "Production of multigigawatt sub-nanosecond microwave pulses by the method of chirpedpulse-amplification," IEEE Electron Device Lett., vol. 42, no. 3, pp. 426-429, 2021, doi: 10.1109/LED.2021.3053131.

[11] L. Zhang, W. He, J. Clarke, K. Ronald, A. D. R. Phelps, and A. W. Cross, "Microwave undulator using a helically corrugated waveguide," IEEE Trans. Electron Devices, vol. 65, no. 12, pp. 5499-5504, 2018, doi: 10.1109/TED.2018.2873726.

[12] S. V. Kuzikov, A. V. Savilov, and A. A. Vikharev, "Flying radio frequency undulator," Appl. Phys. Lett., vol. 105, no. 3, 033504, 2014, doi: $10.1063 / 1.4890586$.

[13] C. R. Donaldson, L. Zhang, M. Beardsley, M. Harris, P. G. Huggard, and W. He, "CNC machined helically corrugated interaction region for a THz gyrotron traveling wave amplifier," IEEE Trans. THz Sci. Technol., vol. 8, no. 1, pp. 85-89, 2017, doi: 10.1109/TTHZ.2017.2778944.

[14] C. R. Donaldson, L. Zhang, M. Harris, M. J. Bearsley, P. G. Huggard, C. G. Whyte, A. W. Cross, and W. He, "8-fold helically corrugated interaction region for high power gyroresonant $\mathrm{THz}$ sources," IEEE Electron Device Lett., 2021, doi: 10.1109/LED.2021.3105435.

[15] S. V. Mishakin, S. V. Samsonov, and G. D. Denisov, "A helicalwaveguide gyro-TWT at the third cyclotron harmonic," IEEE Trans. Electron Devices, vol. 62, no. 10, pp. 3387-3392, 2015, doi: 10.1109/TED.2015.2460265.

[16] C. R. Donaldson, L. Zhang, W. He, A. W. Cross, K. Ronald, and C. G. Whyte, "Low-loss transmission line for a $3.4-\mathrm{kW}, 93-\mathrm{GHz}$ gyrotraveling-wave amplifier," IEEE Trans. Electron Devices, vol. 68, no. 1, pp. 364-368, 2021, doi: 10.1109/TED.2020.3039427.

[17] K. Wang, T. Li, H. Li, H. Wang, B. Hu, Y. Zhou, and C. He, "A broadband $\mathrm{TE}_{01}-\mathrm{TE}_{11}$ mode converter with elliptical section for gyroTWTs," Phys. Rev. Lett., vol. 84, no. 12, pp. 2746-2749, 2000, doi: 10.1103/PhysRevLett.84.2746.

[18] M. H. Honari and R. Mirzavand, "Dielectric-loaded TM $\mathrm{T}_{01}-\mathrm{TE}_{11}$ mode converters for wideband applications," IEEE Trans. Electron Devices, vol. 31, no. 1, pp. 5-8, 2021, doi: 10.1109/LMWC.2020.3034052.

[19] S. Yang and H. Li, "Optimization of novel high-power millimeterwave $\mathrm{TM}_{0} 1 / \mathrm{TE}_{1} 1$ mode converters," IEEE Trans. Micro. Theory Techn. vol. 45, no. 4, pp. 552-554, 1997, doi: 10.1109/22.566638.

[20] L. Zhang, W. He, C. R. Donaldson, G. M. Smith, D. A. Robertson, R. I. Hunter, and A. W. Cross, "Optimization and measurement of a smoothly profiled horn for a W-band gyro-TWA," IEEE Trans. Electron Devices, vol. 64, no. 6, pp. 2665-2669, 2017, doi: 10.1109/TED.2017.2687949.

[21] D. Hass and M. Thumm, "Design procedure for a broadband te ${ }_{11} / \mathrm{he}_{11}$ mode converter for high-power radar applications," J. Infrared Milli. THz Waves, vol. 42, pp. 380-390, 2021, doi: 10.1007/s10762-021-00773-y.

[22] L. Zhang, W. He, K. Ronald, A. D. R. Phelps, C. G. Whyte, C. W. Robertson, A. Y. Young, C. R. Donaldson, and A. W. Cross, "Multimode coupling wave theory for helically corrugated waveguide," IEEE Trans. Microw. Theory Techn., vol. 60, no. 1, pp. 1-7, 2012, doi: 10.1109/TMTT.2011.2170848.

[23] Y. Wang, L. Wang, G. Liu, G. X. Shu, K. Dong, J. X. Wang, R. Yan, H. Fu, Y. L. Yao, Y. Luo, and S. F. Wang, "Wideband circular $\mathrm{TE}_{21}$ and $\mathrm{TE}_{01}$ mode converters with same exciting topologies," IEEE
Trans. Electron Devices, vol. 63, no. 10, pp. 4088-4095, 2016, doi: 10.1109/TED.2016.2596785.

[24] T. H. Chang, C. H. Li, C. M. Wu, and C. F. Yu, "Generating pure circular $\mathrm{TE}_{m n}$ modes using Y-type power dividers," IEEE Trans. Mirow. Theory Techn., vol. 58, no. 6, pp. 1543-1550, 2010, doi: 10.1109/TMTT.2010.2048252. 